\title{
Enteral nutrition in advanced dementia: an unresolved dilemma in clinical practice
}

\author{
Paolo Orlandoni ${ }^{1} \cdot$ Nikolina Jukic Peladic $^{2} \cdot$ Antonio Cherubini $^{3}$
}

Published online: 28 January 2020

(c) European Geriatric Medicine Society 2020

Enteral Nutrition (EN), also named Tube feeding (TF), refers to nutrition therapy where enteral nutrition formula is delivered to gastrointestinal tract of an individual, mostly via nasogastric tube (NGT) or percutaneous endoscopic gastrostomy (PEG). Clinical practitioners' decisions concerning EN in patients with specific clinical conditions are directed by clinical practice guidelines. Clinical guidelines are statements and recommendations based on the best scientific evidence or consensus among experts whose aim is to increase the quality of the care and to decrease the variation in clinical practice by linking the professional services to quality standards [1]. The higher is the strength of the scientific evidence on which each statement and recommendation is based, the higher is the importance of respecting it.

The most relevant recommendations on EN in patients with severe dementia are provided by the American Geriatric Society (AGS) in its Feeding Tubes in Advanced Dementia Position Statement from 2014 and by the European Society for Parenteral and Enteral Nutrition (ESPEN) guidelines on nutrition in dementia from 2015 [2, 3]. Both AGS and ESPEN advise not to initiate EN in patients with severe dementia mostly based on results of studies which showed that EN is not associated with longer survival in these patients [4-15]. Moreover, according to studies cited in the AGS statement, EN does not improve the nutritional

Paolo Orlandoni

p.orlandoni@inrca.it

Nikolina Jukic Peladic

t.nutrizionale@inrca.it; rackog@yahoo.it

Antonio Cherubini

a.cherubini@inrca.it

1 Clinical Nutrition Unit, IRCCS INRCA Ancona, Via Montagnola 81, 60100 Ancona, Italy

2 Vivisol Spa, Via Gerolamo Borgazzi 27, 20900 Monza, Italy

3 Geriatrics, Geriatric Emergency Department and Research Centre on Ageing, IRCCS INRCA Ancona, Via Montagnola 81, 60100 Ancona, Italy status in subjects with severe dementia, it causes excessive use of restraints and it is ineffective in preventing and treating pressure ulcers (PU) and aspiration pneumonia. Despite the recommendations, the use of EN in patients with severe dementia, who always develop swallowing difficulties, is very controversial. Clinicians often do not agree that PEG placement is contraindicated in advanced dementia and they believe that enteral feeding may prevent aspiration pneumonia, weight loss and pressure sores in these subjects. Therefore, EN is still commonly used in clinical practice $[16,17]$. This attitude is supported by the criticism that many authors and scientists expressed about the quality of the scientific evidence used to draft the recommendations $[18,19]$.

Indeed, the studies on which recommendations are based have several weaknesses and new evidence, which suggests that the recommendations against initiating EN in severe dementia should probably be less categorical, has been produced only recently.

The main critics that may be moved to recommendations concerns the methodological limitations of the studies used to draft them. These studies are not prospective randomised controlled clinical trials which are the gold standard for any medical decision but, instead, they are observational studies whose validity is limited. Other important methodological limitations of the studies have been highlighted by Samson and, more recently, by Lynch $[18,20]$. Both authors pointed out that the studies used to draft the recommendations were mostly plagued with selection bias. Clinical outcomes of tube fed patients with advanced dementia were compared with outcomes of patients whose swallowing problems were less severe and who were still able to eat by mouth. The outcomes of different groups of patients were obviously influenced by their different clinical conditions and nutritional regimens and, although some authors attempted to correct this selection bias, the outcomes of different groups are incomparable.

Furthermore, none of the studies provided information on consequences and complications of PEG positioning and on 
their impact on overall outcomes. None of them described the characteristics of follow-up programmes and practices which are unavoidably associated with outcomes of the therapy. The conditions of patients with advanced dementia are severe but these subjects are not necessarily near the end of life so that the outcomes of EN therapy, just as the frequency of EN-related complications and the possibility to resolve them promptly, are strongly dependent on characteristics of the services provided and on the frequency and accuracy of outpatient visits. No information on training of formal and informal caregivers is given either, although it is well known that caregivers have a fundamental role in the management of patients treated with $\mathrm{EN}$ at home or in institutions.

Selection bias and inaccurate methodologies are just two of the numerous issues which impact negatively the quality of the evidence used to draft recommendations on EN in severe dementia.

Low-quality evidence is also available concerning the impact of tube feeding on nutritional status of patients with severe dementia. According to most authors, the EN therapy is inefficient in improving nutritional status in these subjects. Peck is the only author whose study offers different and contrasting results [13]. However, studies used to draft the recommendations were all performed few years ago when the indicators of nutritional status such as albumin levels, the haematocrit and cholesterol levels or some single anthropometric indicators like BMI or weight loss were used [11-14]. Nowadays, the validity of these indicators has been questioned and new evidence should be collected using the proper tools and criteria for the nutritional assessment, such as ESPEN Consensus Statement or GLIM criteria [21, 22].

The argument concerning the restraint use as an inevitable companion of EN also does not find a solid support in the scientific literature $[13,14]$. Data on the frequency of restraints' use in this specific population are scarce, based on small studies and the information is prevalently gathered among old, frail subjects, independent of the condition of being demented [23]. Different authors considered different types of restraints which are commonly used for patients' safety independent of the condition of being treated with EN. Some very important information was scarcely available when guidelines were edited. For example, even though in some countries, the NGTs are used for long-term tube feeding, almost all available information was for patients with PEG. Very incomplete, if any, was also the information on enteral formulas used. Although the overall benefit of EN therapy concerns not only the survival and the improvement of the nutritional status but also the patient's quality of live (QoL), no information on this issue is still available for patients with severe dementia.

Other evidence has been provided only after the publication of the guidelines. Relatively to the issue of PU, there is new evidence demonstrating the ability of special, enriched, formulas to improve PU healing compared with standard formulas [24]. As far as harms of EN are concerned, until very recently, the evidence on the safety of $\mathrm{EN}$, measured in terms of mechanical, gastrointestinal and metabolic complications, was not available for tube-fed patients with severe dementia. Different authors reported on the frequency of complications of EN but they analysed data collected in different populations $[15,25,26]$. Some new evidence was made available on this issue by our study which compared the incidence rates of mechanical, gastrointestinal and metabolic complications in 585 consecutive patients and found no difference between the incidence rates of complications in patients with dementia and patients with similar characteristics but without dementia [27]. New evidence on the correlation between EN and aspiration pneumonia is also available. While until recently, the EN was considered by most authors as a risk factor for aspiration pneumonia in patients with severe dementia $[13,14,28]$, some recent studies showed that, on the contrary, there is no difference in the frequency of aspiration pneumonia among tube-fed patients with severe dementia and tube-fed patients without dementia and that aspiration pneumonia can even be prevented by the means of EN in patients with dementia [27, 29].

Finally, from the ethical standpoint, in recent years, it has been recognised that more attention should also be paid to personal feelings and wishes of patients and caregivers. Family members frequently believe that food and water are basic human needs and should be provided no matter what, and they report that this was also the belief of patient in earlier phases of life. This issue becomes particularly sensitive when patients who are not able to make their healthcare decisions do not have advanced healthcare directive or a surrogate decision maker indicated by power of attorney or court and it is unclear whether the clinicians should decide about artificial nutrition and hydration, just like for other medical treatments [30].

In conclusion, there seems to be reason and the need for a critical revision of the recommendations on EN in patient with advanced dementia. The studies performed in the recent years do not overcome the main methodological limitations which characterised previous studies, but they certainly offer some new evidence which should be considered. Ethical aspects have been recognised as very important to be taken into account during the decision-making process. While no prospective randomised trials will ever be available for the obvious ethical reasons, new, carefully planned, prospective observational studies on larger populations should be performed to clarify the risk benefit ratio of EN in these patients. In our view, data collection and production of new evidence should be strongly recommended if the gap between the clinical practice and scientific recommendations has to be overcome. Solid evidence is also crucial for clinicians to provide objective information to caregivers and 
surrogate decision makers on which to base their ethical decisions. In the meanwhile, the overall decision-making process should be personalised as much as possible and any generalisation should be avoided.

Authors' contribution Conceived the study PO. Supervision PO. Data search and analyses NJP. Wrote the original draft NJP. Edit and comment on manuscript PO, NJP and AC.

Funding This research did not receive any specific grant from funding agencies in the public, commercial, or not-for-profit sectors.

\section{Compliance with ethical standards}

Conflict of interest The authors declare that they have no conflict of interest.

Ethics approval and consent to participate Not applicable.

\section{References}

1. Field MJ, Lohr KN (eds) (1990) Institute of Medicine (US) Committee to Advise the Public Health Service on Clinical Practice Guidelines; clinical practice guidelines: directions for a new program. National Academies Press, Washington (DC)

2. American Geriatrics Society Ethics Committee and Clinical Practice and Models of Care Committee (2014) American geriatrics society feeding tubes in advances dementia position statement. JAGS 62:1590-1593

3. Volkert D, Chourdakis M, Faxen-Irving G, Fruhwald T, Landi F, Suominen MH et al (2014) ESPEN guidelines on nutrition in dementia. Clin Nutr 54:1052-1073

4. Murphy LM, Lipman TO (2003) Percutaneous endoscopic gastrostomy does not prolong survival in patients with dementia. Arch Intern Med 163:1351-1353

5. Callahan CM, Haag KM, Weinberg M, Tierney WM, Buchanan NN, Stump TE et al (2000) Outcomes of percutaneous endoscopic gastrostomy among older adults in a community setting. J Am Geriatr Soc 48:1048-1054

6. Sanders DS, Carter MJ, D'Silva J, James G, Bolton RP, Bardhan KD (2000) Survival analyses in percutaneous endoscopic gastrostomy feeding: a worse outcome in patients with dementia. Am J Gastroenterol 95:1472-1475

7. Abuksis G, Mor M, Segal N, Shemesh I, Plout S, Sulkes J et al (2000) Percutaneous endoscopic gastrostomy: high mortality rates in hospitalized patients. Am J Gastroenterol 95:128-132

8. Mitchell SL, Kiely DK, Lipsitz LA (1997) The risk factors and impact on survival of feeding tube placement in nursing home residents with severe cognitive impairement. Arch Intern Med 157:327-332

9. Meier DE, Ahronheim JC, Morris J, Baskin-Lyons S, Morrison RS (2001) High short-term mortality in hospitalized patients with advanced dementia: lack of benefit of tube feeding. Arch Intern Med 161:594-599

10. Nair S, Hertan H, Pitchumoni CG (2000) Hypoalbuminemia is a poor predictor of survival after percutaneous endoscopic gastrostomy in elderly patients with dementia. Am J Gastroenterol 95:133-136
11. Alvarez- Fernandez B, Garcia-Ordonez MA, Martinez-Manzanares C, Gomez-Huelgaz R (2005) Survival of a cohort of elderly patients with advanced dementia: nasogastric tube feeding as a risk factor for mortality. Int J Geriatr Psychiatry 20:363-370

12. Jaul E, Singer P, Calderon-Margalit R (2006) Tube feeding in the demented elderly with severe disabilities. Isr Med Assoc J 8:870-874

13. Peck A, Cohen CE, Mulvihill MN (1990) Long-term enteral feeding of aged demented nursing home patients. J Am Geriatr Soc 38:1195-1198

14. Teno JM, Gozalo PL, Mitchell SL, Kuo S, Rhodes RL, Bynum JP et al (2012) Does feeding tube insertion and its timing improve survival? J Am Geritar Soc 60:1918-1921

15. Kuo S, Rhodes RL, Mitchell SL et al (2009) Natural history of feeding tube use in nursing home residents with advanced dementia. J Am Med Dir Assoc 10:264-270

16. Fessler TA, Short TB, Willcutts KF, Sawyer RG (2019) Physician opinions on decision making for percutaneous endoscopic gastrostomy (PEG) feeding tube placement. Surg Endosc. https:// doi.org/10.1007/s00464-019-06711-3

17. Punchik B, Komissarov E, Zeldez V, Freud T, Samson T, Press Y (2018) Doctors' knowledge and attitudes regarding enteral feeding and eating problems in advanced dementia. Dement Geriatr Cogn Dis Extra 8(2):268-276. https://doi.org/10.1159/000489489

18. Lynch MC (2016) Is tube feeding futile in dementia. Linacle $Q$ 83(3):283-307

19. Regnard C et al (2010) Gastrostomies in dementia: bad practice or bad evidence. Age Aging 39:282

20. Sampson EL, Candy B, Jones L (2009) Enteral tube feeding for older people with advanced dementia. Cochrane Database Syst Rev 2009:1-25

21. Cederholm T, Bosaeus I, Barazzoni R, Bauer JA, Klek S et al (2015) Diagnostic criteria for malnutrition: an ESPEN consensus statement. Clin Nutr 34(3):335-340

22. Jensen GL, Cederholm T, Correi MITD, Gonzales MC, Fukushima R, Higashiguchi T et al (2019) GLIM criteria for the diagnosis of malnutrition: a consensus report from the global clinical nutrition community. JPEN 43(1):32-40

23. Kvale E, Dionne-Odom JN, Redden DT, Bailey FA, Bakitas M, Goode PS et al (2015) Predictors of physical restraint use in hospitalized veterans at end of life: an analysis of data from the BEACON trial. J Palliat Med 18(6):520-526. https://doi.org/10.1089/ jpm.2014.0354

24. Cereda E, Neyens JCL, Caccialanza R, Rondanelli M, Schols JMGA (2017) Efficacy of a disease-specific nutritional support for pressure ulcer healing: a systematic review and meta-analysis. J Nutr Health Aging 21(6):655-661. https://doi.org/10.1007/s1260 3-016-0822-y

25. Hull MA, Rawlings SRD, Field RGN, Allison SP, Murray FE, McIntyre AS et al (1993) Audit of outcome of long-term enteral nutrition by percutaneous endoscopic gastrostomy. Lancet 341(8849):869-872

26. Rimon E, Kagansky M, Levy S (2005) Percutaneous endoscopic gastrostomy: evidence of different prognosis in various patient subgroups. Age Ageing 34:353-357. https://doi.org/10.1093/agein g/afi085

27. Orlandoni P, Jukic-Peladic N, DiRosa M et al (2019) The outcomes of long term home enteral nutrition (HEN) in older patients with severe dementia. Clin Nutr 38(4):1871-1876. https://doi. org/10.1016/j.clnu.2018.07.010

28. Ciocon JO, Silverstone FA, Graver LM et al (1988) Tube feeding in elderly patients. Indications, benefits, and complications. Arch Intern Med 148:429-433

29. Takenoshita S, Kondo K, Okazaki K, Hirao A, Takayama K, Hirayama K et al (2017) Tube feeding decreases pneumonia rate in patients with severe dementia: comparison between pre- and 
post-intervention. BMC Geriatr. https://doi.org/10.1186/s1287 7-017-0662-6

30. Shintani S (2013) Efficacy and ethics of artificial nutrition in patients with neurologic impairments in home care. J Clin Neurosci 20:220-223
Publisher's Note Springer Nature remains neutral with regard to jurisdictional claims in published maps and institutional affiliations. 